\title{
ON SOME EXTREMALITIES IN THE APPROXIMATE INTEGRATION
}

\author{
SZYMON WĄSOWICZ
}

Abstract. Some extremalities for quadrature operators are proved for convex functions of higher order. Such results are known in the numerical analysis, however they are often proved under suitable differentiability assumptions. In our considerations we do not use any other assumptions apart from higher order convexity itself. The obtained inequalities refine the inequalities of Hadamard type. They are applied to give error bounds of quadrature operators under the assumptions weaker from the commonly used.

Mathematics subject classification (2010): 26D15, 41A55, 41A80, 26A51, 65D30, 65D32.

Keywords and phrases: Approximate integration, error bounds, extremalities, Hadamard-type inequalities, higher order convexity, quadrature rules, support theorems.

\section{REFERENCES}

[1] M. BessenyeI, Zs. PÁLES, Higher-order generalizations of Hadamard's inequality, Publ. Math. Debrecen 61 (2002), 623-643.

[2] B. D. Bojanov, P. P. Petrov, Gaussian interval quadrature formula, Numer. Math. 87 (2001), 625-643.

[3] H. BRASS, K. PETRAS, Quadrature theory, online: http://www-public.tu-bs.de:8080/ $\sim$ petras/book/qt2.ps

[4] H. BRASS, G. SCHMEISSER, Error estimates for interpolatory quadrature formulae, Numer. Math. 37 (1981), 371-386.

[5] P. S. Bullen, A criterion for $n$-convexity, Pacific J. Math. 36 (1971), 81-98.

[6] F. B. HildeBRAND, Introduction to Numerical Analysis, McGraw-Hill Book Company, New York 1956.

[7] E. HopF, Über die Zusammenhänge zwischen gewissen höheren Differenzenquotienten reeller Funktionen einer reellen Variablen und deren Differenzierbarkeitseigenschaften, Dissertation, Friedrich-WilhelmsUniversität Berlin, 1926.

[8] S. KARLIN, W. J. STUDDEN, Tchebycheff systems: with applications in analysis and statistics, Interscience Publishers, New York 1966.

[9] M. KuCZMA, An Introduction to the Theory of Functional Equations and Inequalities. Cauchy's Equation and Jensen's Inequality, Państwowe Wydawnictwo Naukowe (Polish Scientific Publishers) and Uniwersytet Śląski, Warszawa-Kraków-Katowice 1985.

[10] A. Pinkus, D. WulBert, Extending n-convex functions, Studia Math. 171 (2005), 125-152.

[11] T. Popoviciu, Sur quelques propriétés des fonctions d'une ou de deux variables réelles, Mathematica (Cluj) 8 (1934), 1-85.

[12] A. Ralston, A first course in numerical analysis, McGraw-Hill Book Company, New York, St. Louis, San Francisco, Toronto, London, Sydney, 1965.

[13] A. W. Roberts, D. E. Varberg, Convex Functions, Academic Press, New York 1973.

[14] G. SzEGő, Orthogonal Polynomials, American Math. Society, Providence, Rhode Island, 1939.

[15] S. WĄSOWICZ, Hermite-Hadamard-type inequalities in the approximate integration, Math. Inequal. Appl., 11(4) (2008), 693-700.

[16] S. WĄSOWICZ, Inequalities between the quadrature operators and error bounds of quadrature rules, J. Ineq. Pure \& Appl. Math. (JIPAM), 8 (2007), Article 42, online: http: // jipam . vu . edu . au

[17] S. WĄSOWICZ, On error bounds for Gauss-Legendre and Lobatto quadrature rules, J. Ineq. Pure \& Appl. Math. (JIPAM) 7 (2006), Article 84, online: http://jipam.vu.edu . au

[18] S. WĄSOWICZ, On quadrature rules, inequalities and error bounds, J. Ineq. Pure \& Appl. Math. (JIPAM), 9 (2008), Article 36, online: http: // jipam. vu.edu. au 
[19] S. WĄSOWICZ, Support-type properties of convex functions of higher order and Hadamard-type inequalities, J. Math. Anal. Appl. 332 (2007), 1229-1241.

[20] E. W. WeISSTEIN, Legendre-Gauss quadrature, from MathWorld-A Wolfram Web Resource, online: http://mathworld.wolfram.com/Legendre-GaussQuadrature.html

[21] E. W. WeISSTEIN, Lobatto quadrature, from MathWorld-A Wolfram Web Resource, online: http://mathworld.wolfram.com/LobattoQuadrature.html

[22] E. W. WeIsSTEIN, Radau quadrature, from MathWorld-A Wolfram Web Resource, online: http://mathworld.wolfram.com/RadauQuadrature.html 